\title{
The Occlusal Plane Inclination Analysis for Determining Skeletal Class III Malocclusion Diagnosis
}

This article was published in the following Dove Press journal: Clinical, Cosmetic and Investigational Dentistry

\section{Gusti Aju Wahju Ardani id Ageng Wicaksono (D) Thalca Hamid (iD \\ Orthodontics Department, Faculty of Dental Medicine, Universitas Airlangga, Surabaya, East Java, Indonesia}

Introduction: The occlusal plane is very important in the stomatognathic and aesthetic dentofacial system. Manipulation of the occlusal plane plays an important role in class III malocclusion correction. For this reason, the strategy in establishing the diagnosis, prognosis and treatment plan becomes more complex, so a more comprehensive analysis of the occlusal plane inclination as a parameter of diagnosis is needed for more effective and efficient treatments.

Aim: The aim of this study was to determine the comparison of occlusal plane inclination between class III and class I malocclusions and to knowing the correlation of occlusal plane inclination in class III malocclusions with the direction of facial growth.

Materials and Methods: Samples were secondary lateral cephalograms collected at the Universitas Airlangga (UNAIR) Dental Hospital in October 2017-2018. Cephalograms were traced using the Orthovision ${ }^{\mathrm{TM}}$ software; then, the Mann-Whitney comparative statistical test $(\mathrm{p}<0.05)$ was carried out followed by the canonical correlation test.

Results: Differences in class III and class I malocclusions were found in the OP-AB and $\mathrm{OP}-\mathrm{FH}$ angles. The OP-AB angle is the occlusal plane inclination which has the strongest correlation compared to others. $\mathrm{OP}-\mathrm{AB}$ angle is strongly correlated with horizontal growth parameter, especially theSNB. The OP-AB angle is also correlated with vertical growth parameters: the $\mathrm{Y}$ axis and the Facial axis.

Conclusion: The $\mathrm{OP}-\mathrm{AB}$ angle is a parameter that can distinguish between class III and class I malocclusions. The OP-AB angle has a strong correlation with vertical and horizontal growth directions.

Keywords: cephalometry, facial growth, class III malocclusion, occlusal plane

\section{Introduction}

Skeletal class III malocclusion is one of the most difficult problems faced by orthodontists. ${ }^{1}$ Aesthetics is always the primary complaint in patients with class III malocclusions. Skeletal class III malocclusion is caused by undergrowth of the maxilla, overgrowth of the mandible, or a combination of both. ${ }^{1,2}$ The highest prevalence of class III malocclusion in the population of East Asia $16.59 \%$ and Southeast Asia (Javanese ethnic included) $15.69 \%{ }^{3}$

Patients with class III malocclusion, the mandibular first molar relation is more mesial than normal, sometimes also accompanied by mismatching the width of the upper and lower jaw arches. The more older the more severe degree of malocclusion. ${ }^{4}$ During the dentoskeletal growth phase unilaterally decreasing vertical height causes
Correspondence: I Gusti Aju Wahju

Ardani

Department of Orthodontics, Faculty of Dental Medicine, Universitas Airlangga, J. Prof. Dr. Moestopo 47, Surabaya, Indonesia

Tel/Fax +62 315030255

Email wahju_ardani@fkg.unair.ac.id 
the position of the mandible to deviate in the same direction. This results in lateral displacement of the condilar during functional movements, including opening and closing the mouth. Contralateral differences in vertical dimensions can produce masticatory muscle imbalances that cause mandibular asymmetry and disruption of the temporomandibular joints. ${ }^{4,5}$ Patients with class III malocclusion have different facial patterns depending on the inclination of the occlusal plane. Manipulation of the occlusal plane plays an important role to get better treatment results. ${ }^{6}$

The occlusal plane is very important in the stomatognathic and aesthetic dentofacial system. The shape and slope (morphology and inclination) of the occlusal plane holds individual inclination. The inclination of the occlusal plane is an important factor in both static occlusion and dynamic occlusion in relation to masticatory movements. The occlusal plane angle also affects the smile especially on the incisal smile arch of the maxillary incisors and the tip of the canine. ${ }^{5}$ Inclination in the occlusal plane becomes an important parameter for obtaining harmonious orofacial relations. ${ }^{6}$

The use of orthodontic devices such as reverse headgear, chin cap, and functional devices used early can prevent the severity of malocclusion. But in some cases patients who have passed the growth spurt phase are less satisfying. So that further therapy is needed in the form of camouflage orthodontics or even surgical orthodontics. In the case of borderline class III malocclusion, more surgical results provide good and stable results, but for economic reasons, psychology and a large risk factor, surgery is less accepted by the public. ${ }^{4}$ At present the use of orthodontic mini implants or temporary anchorage devices (TAD) is increasing dramatically. The benefits of using TAD can provide absolute anchoring, facilitate treatment and reduce complaints from patients. ${ }^{7,8}$

Camouflage orthodontic correction using elastic class III, the occlusal plane angle will decreases and the Witts number will increase, mandibular rotation going down and back. In class III malocclusions of the facial type with a low occlusal plane clockwise rotation of the mandible would be very beneficial but not in the high occlusal plane facial type, therefore optimal anchoring is very necessary. ${ }^{6,8}$

Along with technological developments, camouflage orthodontic treatment is making rapid progress. So that the paradigm has shifted, which previously had to use surgical orthodontics, now some conditions can be corrected with camouflage orthodontics. For this reason, the strategy in establishing the diagnosis, prognosis and treatment plan becomes more complex, so a more comprehensive analysis of the occlusal plane inclination as a parameter of diagnosis is needed for more effective and efficient treatments. ${ }^{7,8}$ Thus, the aim of this study was to determine the comparison of occlusal plane inclination between class III and class I malocclusions and to knowing the correlation of occlusal plane inclination in class III malocclusions with the direction of facial growth.

\section{Materials and Methods Sample}

The study was performed in accordance with the Declaration of Helsinki, and human ethics approval was attained from the Faculty of Dental Medicine, Universitas Airlangga, Health Research Ethical Clearance Commission with number 716/ HRECC.FODM/X/2019. Samples were secondary cephalometric radiographic film collected at the Universitas Airlangga (UNAIR) Dental Hospital in October 2017-2018. All patients provided a written informed consent to review their medical records as per the policy of Faculty of Dental Medicine, UNAIR. The confidentiality of the patient's identity remains protected and for patients under 17 years old the informed consent were represented by their parents. From the total sample, there were 248 patients with skeletal Class I malocclusion (ANB 1-4), and 46 patients with skeletal Class III malocclusion (ANB $<0$ ). There were only 40 skeletal Class III malocclusion patients who meet our eligibility criteria, with the age range of 15-35 years. The Inclusion criteria of sample selection were ANB $<0$, male or female patient with skeletal Class III malocclusion who had never done any orthodontics treatment before full permanent dentitions. Then the study data meet the criteria compared to the inclination of the occlusal plane based on class I and class III malocclusions.

The cephalograms of patients with ANB less than $0^{\circ}$ were included in the class III malocclusion sample group, whereas the cephalograms of patients with ANB $0^{\circ}-4^{\circ}$ were included in the class I malocclusion sample group. Cephalometry tracing is carried out twice with a lag period of 2 weeks by the same operator. The cephalogram was traced using the Orthovision $^{\mathrm{TM}}$ digital program and then the anatomical points are determined as shown in Figures 1-3.The direction of growth of the nasomaxilla and mandible complex is divided into vertical and horizontal directions.

Growth of the nasomaxillary and mandibular complexes in the vertical direction is measured using the following cephalometric parameters (Figure 1):

(a) Facial Axis: The angle between Pt-Gn and cranial axis (Ba-Na)

(b) Y Axis: The angle between S-Gn and the FH plane 


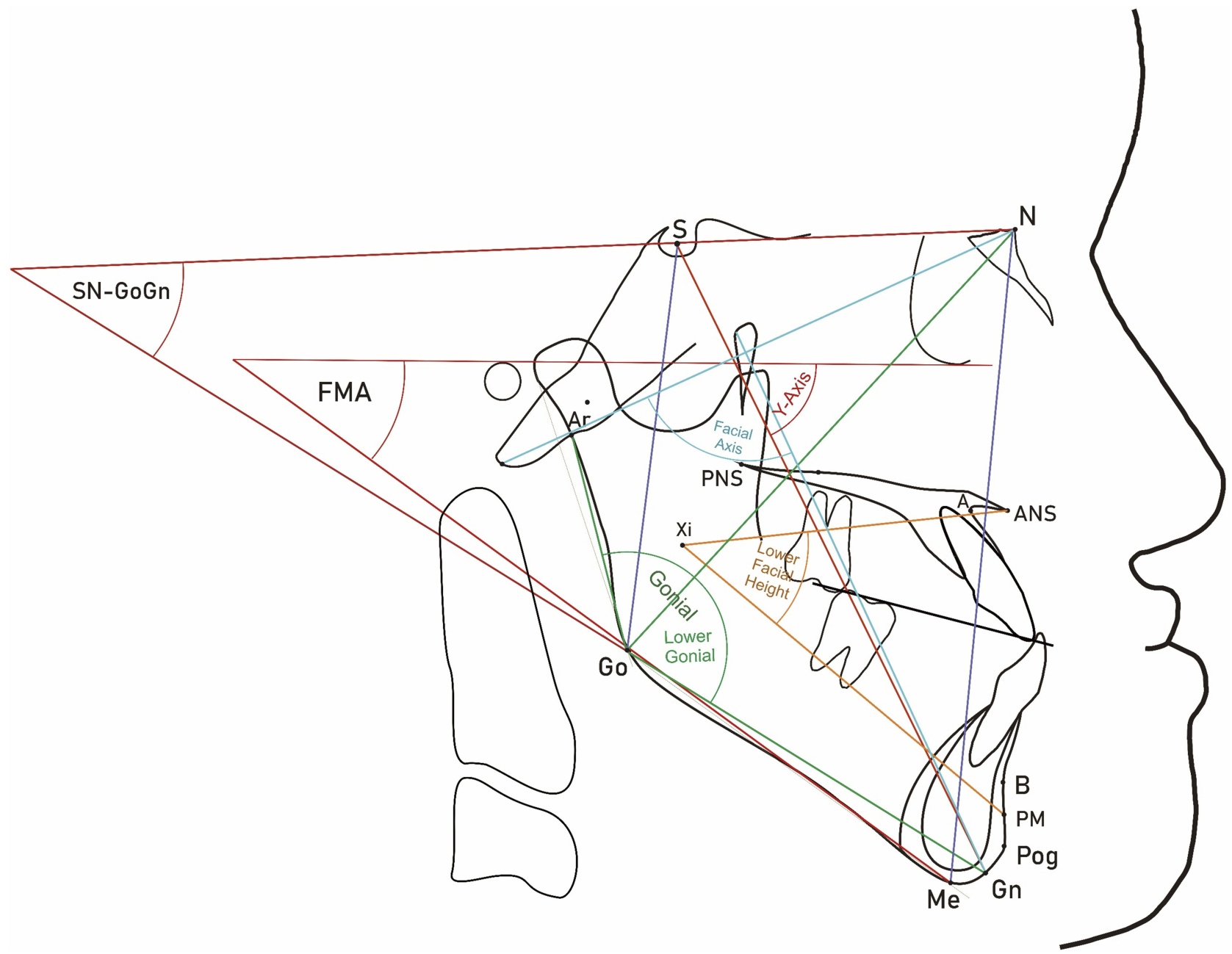

Figure I Anatomical lines and angles used in this study to determine vertical dysplasia and growth of the nasomaxillary and mandibular complexes in the vertical direction.

(c) FMA: The angle between the FH plane and the mandible plane

(d) SN-GoGn: The angle between the SN plane and the Go-Gn line

(e) Gonial Angle: The angle between the Go-Gn line and the Go-Ar line

(f) Lower Facial height: The angle between the ANSXi line and the PmXi line (Rickets)

(g) Lower Gonial Angle: The angle between the GoGn line and the Go-N line

Growth of the nasomaxillary and mandibular complexes in the horizontal direction was measured using the following cephalometric parameters (Figure 2):

(a) SNA: The angle between the cranii base $(\mathrm{SN})$ and the NA line (b) SNB: The angle between the cranii base $(\mathrm{SN})$ and the NB line

(c) ANB: The angle between the NA and NB lines

(d) Saddle Angle: The angle between the SN plane and the S-Ar line

(e) Upper Gonial Angle: The angle between the Go-N line and the Go-Ar line

The inclination of the occlusal plane are the inclination parameters of the occlusal plane in relation to the nasomaxillary and mandibular complexes, measured using the cephalometric angle parameters as follows (Figure 3):

(a) OP-SN: The angle between the cranii base (SN plane) and the occlusal plane

(b) OP-FH: The angle between the FH plane and the occlusal plane 


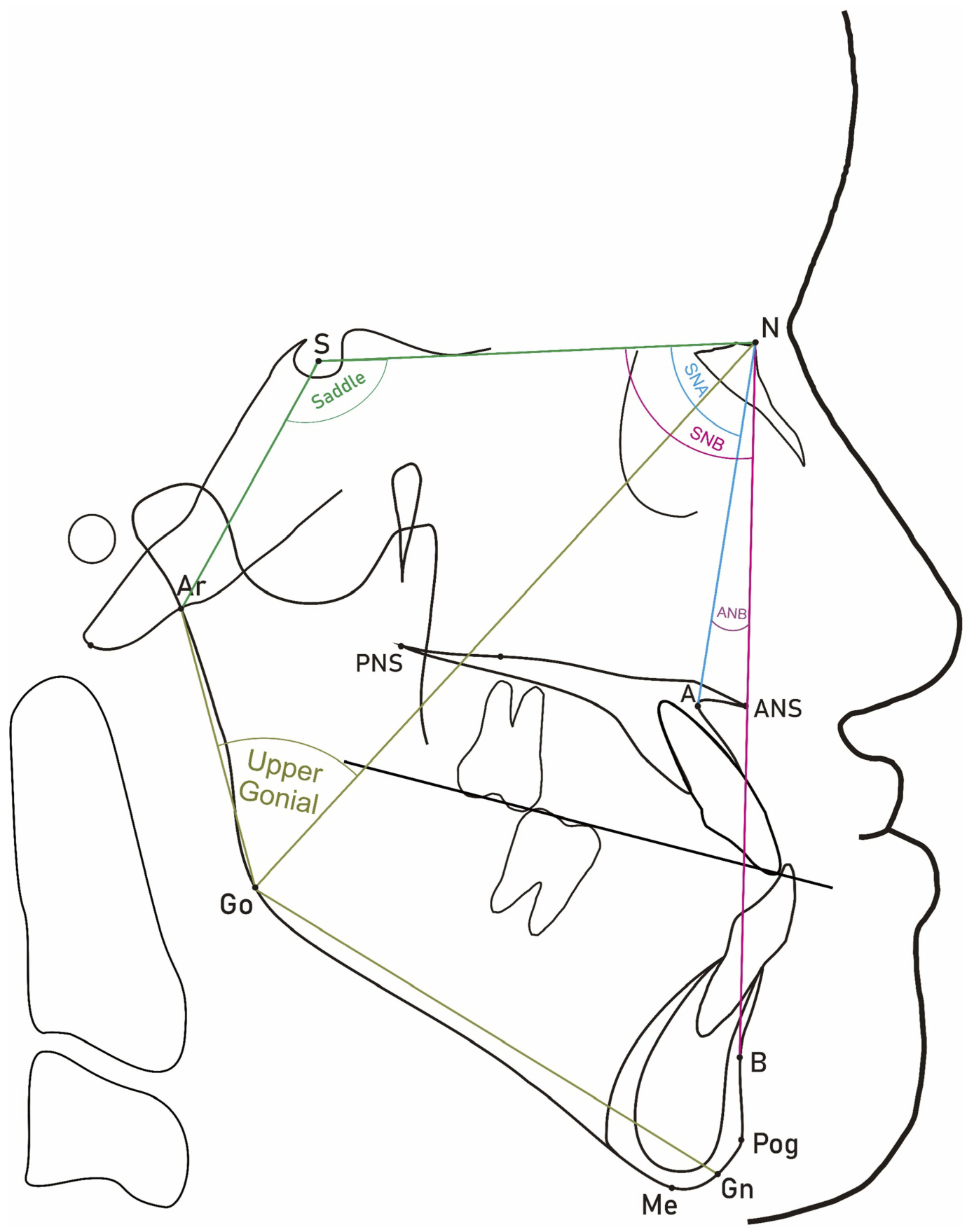

Figure 2 Anatomical lines and angles used in this study to determine sagittal dysplasia and growth of the nasomaxillary and mandibular complexes in the horizontal direction.

(c) OP-GoGn: The angle between the mandibular plane (Go-Gn) and the occlusal plane

(d) OP-AB: The angle between the AB plane and the occlusal plane

\section{Statistical Analysis}

Statistical analysis were using Statistical Package for Social Science (SPSS) version 25 (IBM Corporation, Illinois, US).
Cohen's Kappa test was conducted to determine the reliability of the measurement data. The statistical analysis used to determine the differences between the inclination of occlusal planes in class I and class III malocclusions is the Mann-Whitney test comparative analysis $(p<0.05)$. To determine the distribution of data using the one-sample Kolmogorov-Smirnov test $(p>0.05)$. Statistical analysis used to determine the correlation between the direction of 


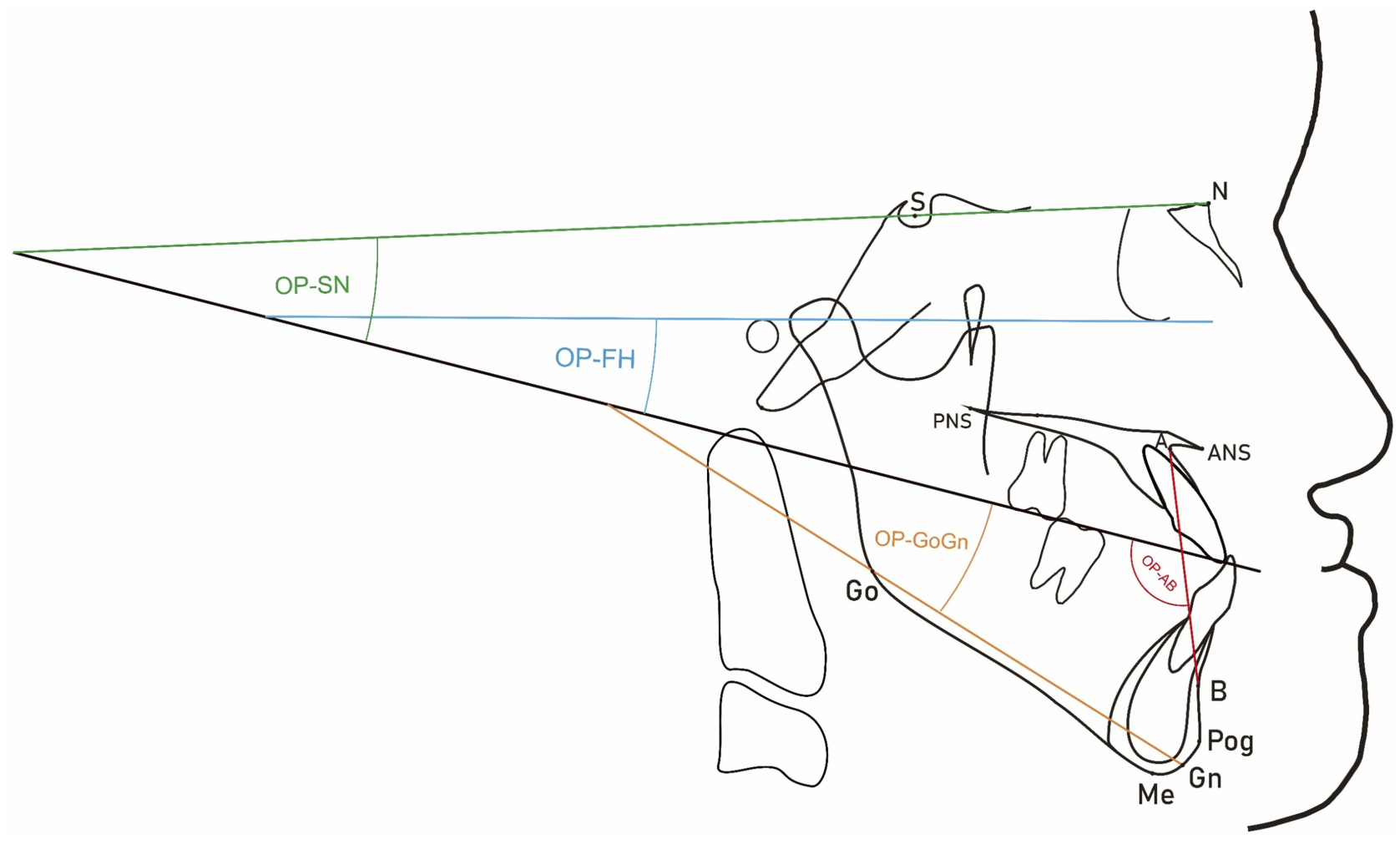

Figure 3 Anatomical lines and angles used in this study to determine the inclination of the occlusal plane.

development of nasomaxillary and mandibular complexes with the inclination of occlusal planes used statistical analysis of canonical correlation ( $p>0.05$ ).

\section{Results}

The Kolmogorov-Smirnov normality test results for class I and class III malocclusion data indicate that the data are not normally distributed $(\mathrm{p}<0.05)$. The normality test results for class III malocclusion data indicate that the data are normally distributed $(\mathrm{p}>0.05)$.

Descriptive statistics of class I can be seen that the average parameters that exceed normal values are Facial Axis (103.40), Lower Gonial Angle (78.77), OP-FH (13.15) and OP-GoGn (20.29). Descriptive statistics of class III mean parameters that exceed normal values are Gonial Angle (130.15), Upper Gonial Angle (51.12), Lower Gonial Angle (79.04), SNB (85.23), OP-FH (14.25) and OP-AB (108.92) (Table 1).

There was a difference between class I and class III malocclusions. The significance in this study for the OP$\mathrm{SN}$ angle and OP-GoGn angle. There was no difference between the class I and class III malocclusion groups. The significance for the $\mathrm{OP}-\mathrm{FH}$ and $\mathrm{OP}-\mathrm{AB}$ angles meaning that there is a difference between the class I and class III malocclusion groups (Table 2).

Canonical loading is also referred to as structure correlation, measuring simple linear correlations between observational data in the independent variable or the dependent variable with its canonical variable collection. The origin variable that has a large canonical load value ( $p>0.5$ ) will be said to have a large role in the collection of variables. The canonical loading sign indicates the direction of the relationship. The greater the value of the canonical load, the more important the role of the origin variable will be in the variable set.

Interpretation of canonical loads from the results of the study:

The first canonical function the sequence of contributions relative to the angle dependent variable OP-AB (0.656) correlates with horizontal growth parameters namely ANB (-0.767) and SNB (0.746). The OP-AB angle also correlates with the vertical growth parameters, the $\mathrm{Y}$ axis $(-0.662)$, and the Facial axis $(-0.599)$ (Table 3$)$.

The second canonical function the sequence of contributions relative to the angle dependent variable OP-FH angle (0.794) and OP-AB (0.708) correlates with the vertical growth parameters namely Lower Gonial Angle 
Table I Descriptive Data of Measurement Class III Malocclusions Variable

\begin{tabular}{|l|l|l|l|l|l|}
\hline & Minimum & Maximum & Mean & Std. Dev & Normal Value \\
\hline Saddle Angle & 113.75 & 134.17 & 123.33 & 4.73 & $119.20-128.60$ \\
Facial Axis & 76.43 & 100.03 & 89.15 & 4.87 & $82.74-90$ \\
Y-Axis & 52.64 & 73.24 & 62.41 & 5.21 & $53-64.6$ \\
FMA & 17.51 & 45.26 & 31.18 & 7.37 & $16-35$ \\
SN-GoGn & 20.51 & 48.74 & 34.11 & 7.06 & $24.82-35.76$ \\
Gonial Angle & 111.23 & 150.13 & 130.15 & 8.86 & $110.32-123.0$ \\
UPper Gonial Angle & 42.41 & 64.02 & 51.12 & 5.00 & $40.30-47.70$ \\
Lower Gonial Angle & 66.28 & 89.38 & 79.04 & 6.54 & $68.5-77.58$ \\
Byork Sum & 380.79 & 408.74 & 394.79 & 7.18 & $336.20-395.6$ \\
SNA & 71.64 & 86.32 & 80.77 & 3.52 & $79.2-85.6$ \\
SNB & 75.11 & 92.45 & 85.23 & 4.30 & $77.3-83.5$ \\
ANB & -11.01 & -0.42 & -4.47 & 2.74 & $0.30-3.70$ \\
OP-SN & $5.8 I$ & 43.63 & 18.26 & 6.75 & $11.31-19.17$ \\
OP-FH & 2.53 & 26.03 & 14.25 & 6.48 & $5.50-12.1$ \\
OP-GoGn & 5.68 & 28.38 & 16.93 & $12-20$ \\
OP-AB & 99.09 & 123.04 & 65.92 & 6.17 & $88.6-95.8$ \\
Facial Height Ratio & 55.92 & 77.72 & 44.73 & $65.56-74.92$ \\
Lower Facial Height & 30.22 & 58.02 & 5.62 & $42.77-49.79$ \\
\hline
\end{tabular}

Table 2 The Differences in Occlusal Plane Inclination Between Class I and Class III Malocclusions

\begin{tabular}{|l|l|l|l|l|}
\hline Parameter & OP-SN & OP-FH & OP-GoGn & OP-AB \\
\hline Means Class I & 40.60 & 31.70 & 37.38 & 21.15 \\
Means Class III & 40.40 & 49.30 & 43.63 & 59.85 \\
Significance & 0.968 & $0.000^{*}$ & 0.215 & $0.000^{*}$ \\
\hline
\end{tabular}

Note: *Significant at $\mathrm{p}<0.05$.

Table 3 Results of the First Canonical Correlation Set

\begin{tabular}{|l|l|l|l|l|}
\hline \multicolumn{5}{|l|}{ Canonical Loadings } \\
\hline Variables & $\mathbf{I}$ & $\mathbf{2}$ & $\mathbf{3}$ & $\mathbf{4}$ \\
\hline Saddle angle & $0.06 \mathrm{I}$ & -0.425 & $-0.47 \mathrm{I}$ & -0.133 \\
Facial axis & -0.599 & 0.583 & -0.277 & 0.047 \\
Y axis & -0.662 & 0.644 & 0.104 & -0.148 \\
FMA & -0.371 & 0.860 & -0.092 & 0.106 \\
SN-GoGn & -0.425 & 0.576 & -0.586 & 0.234 \\
Gonial angle & 0.049 & 0.741 & 0.023 & 0.394 \\
Uppergonial & 0.286 & 0.355 & 0.277 & 0.285 \\
Lowergonial & -0.174 & 0.836 & -0.208 & 0.359 \\
Byork sum & -0.313 & 0.601 & -0.559 & 0.170 \\
SNA & 0.078 & -0.294 & 0.470 & 0.429 \\
SNB & 0.746 & 0.331 & 0.426 & 0.122 \\
ANB & -0.767 & -0.569 & -0.132 & 0.165 \\
Lower Facial Height & 0.228 & -0.408 & 0.167 & -0.309 \\
Facial Height Ratio & 0.207 & -0.563 & 0.654 & -0.085 \\
\hline
\end{tabular}

(0.836), Gonial angle (0.741), Y axis (0.644), Byork sum (0.601), Facial axis (0.583), SNGoGn (0.576) and Facial Height Ratio (-0.563). The OP-FH and OP-AB angles also correlate with the horizontal growth parameters, ANB $(-0.569)$ (Table 4).

\section{Discussion}

This study focuses on discussing the inclination of the occlusal plane in class III malocclusions in Javanese ethnic at UNAIR Dental Hospital Surabaya, East Java, Indonesia. The inclination of occlusal planes in class III malocclusions are compared with class I malocclusions because they are a differential diagnosis. ${ }^{8}$ The inclination of the occlusal planes studied are the angle of OP-FH, OP-SN, OP-GoGn and OP-AB because they can determine the inclination of the occlusal plane. ${ }^{5,9-11}$ The classification of malocclusion in this study is based on anteroposterior skeletal discrepancy. Anteroposterior discrepancy is the ANB angle which is the difference between the cranial base and apical base of the maxilla (SNA) with the apical base of the lower jaw (SNB). ${ }^{12-14}$

Table 4 Results of the Second Canonical Correlation Set

\begin{tabular}{|l|l|l|l|l|}
\hline \multicolumn{5}{|l|}{ Canonical Loadings } \\
\hline Variables & $\mathbf{I}$ & $\mathbf{2}$ & $\mathbf{3}$ & $\mathbf{4}$ \\
\hline OP-SN & -0.289 & 0.394 & -0.628 & -0.605 \\
OP-FH & -0.262 & 0.794 & $0.1 \mathrm{II}$ & -0.537 \\
OP-GoGn & -0.268 & 0.499 & -0.212 & 0.797 \\
OP-AB & 0.656 & 0.708 & 0.077 & -0.250 \\
\hline
\end{tabular}


This study also discusses the closeness of the correlation inclination of the occlusal plane with other parameters incorporated in the gnathology system. The gnathology system consists of three elements namely teeth, bones and muscles. The teeth are represented by the inclination of the occlusal plane (angle of OP-FH, OP-SN, OP-GoGn and $\mathrm{OP}-\mathrm{AB})$. Bone is represented by the direction of growth of the nasomaxillofacial and mandibular complexes from the vertical direction (FMA, SN-GoGn, Lower Gonial Angle, Facial Height Ratio, Facial Axis, Y Axis, and Byork Sum) and horizontal direction (SNA, SNB, ANB, Saddle Angle and Upper Gonial Angle). ${ }^{10-13}$ Muscle is represented by Lower Facial Height which represents the musculature anchorage. ${ }^{15}$

In this study found that, the comparison of class III and class I malocclusions in the OP-SN and OP-GoGn angle occlusal plane inclination does not have a statistically significant difference. While the comparison of class III and class I malocclusions on the occlusal planes inclination of the OP-FH and OP-AB angle has statistically significant differences. According to Zweig and Campbell, diagnostic indicators must be used to distinguish the presence or absence of abnormalities. ${ }^{16}$ The OP-FH and OP-AB angles differ in class III and class I malocclusions so that they may be considered as indicators of diagnosis. According to Kim and Vietas, dentofacial complex variations are rarely caused by a single factor and therefore consideration of indicators of sagittal dysplasia diagnosis is not single and absolute. $^{16}$

In this study, we found that the OP-SN angle in class III samples in the normal range. Based on the third variable canonical correlation test, the OP-SN angle correlates with the vertical growth parameters namely Facial Height Ratio, SN-GoGn and Byork sum. The greater the value of Facial Height Ratio means that the height of the anterior face is shorter than the height of the posterior face, the smaller the OP-SN angle. The greater the $\mathrm{SN}-\mathrm{GoGn}$ and Byork sum (the more hyperdivergence), the greater the OP-SN angle.

According to Ellis and Mc Namara, the SN plane gives less accurate results than the Frankfurt Horizontal plane with respect to the position of the jaw. ${ }^{14}$ Hopkin and Houston state that the SN plane representing the anterior cranial base lacks a strong relationship with the dental arch and jaw. ${ }^{17}$ The SN plane is used to determine hyperdivergence, whereas hyperdivergence can occur in both class I and class III malocclusions. ${ }^{14,17}$ Therefore the OP-SN angle does not have a difference between class III and class I malocclusions.

We found that OP-GoGn angle in class III samples are still in the normal range. Schudy argues that the angle of OP-GoGn determines the prognosis for treatment of vertical dysplasia. The OP-GoGn angle is one of the parameters in determining skeletal divergence patterns and is not affected by sagittal dysplasia, ${ }^{14}$ so that the OP-GoGn angle between class III and class I malocclusions is no significant difference.

In this study, we found that the OP-FH angle in class III sample shave normal range. This shows the rotation of the occlusal plane clockwise. Based on the second variable canonical correlation test, the OP-FH angle correlates with the vertical growth parameters namely Lower Gonial Angle, Gonial angle, Y axis, Byork sum, Facial axis, SNGoGn and Facial Height Ratio. The OP-FH angle is also correlated with the horizontal growth parameter, ANB. The greater the hyperdivergence and the smaller the anteroposterior discrepancy of the jaw, the greater the angle of OP-FH. The Frankfurt plane gives more accurate results on the jaw position than other horizontal facial plane because the position is closer to the median, ${ }^{14,18}$ therefore the OP-FH angle is significantly different between class III and class I malocclusion samples.

In our study result we found that the OP-AB angle in class III samples have above the normal range. This indicates the clockwise rotation of the occlusal plane or the counter-clockwise rotation of the AB plane. The OP-AB angle is a characteristic of the occlusal plane whose angles are obtained from two planes that intersect almost perpendicularly. Point $\mathrm{A}$ and point $\mathrm{B}$ are known as sagittal dysplasia parameters, ${ }^{14,18}$ so OP-AB can distinguish sagittal dysplasia.

Based on the first variable canonical correlation test, the $\mathrm{OP}-\mathrm{AB}$ angle correlates with the horizontal growth parameters namely ANB and SNB. The OPAB angle is also correlated with vertical growth parameters, the $\mathrm{Y}$ axis, and the Facial axis. The greater the SNB and the smaller $\mathrm{ANB}$, the greater the angle of OP-AB. The greater the angle of the $\mathrm{Y}$ axis and Facial axis, the smaller the angle of the OP-AB. The $\mathrm{Y}$ axis and Facial axis are indicators of the presence of chin rotation relative to the upper face, therefore the OP-AB angle can describe sagittal dysplasia with or without chin rotation. ${ }^{14}$

The direction of vertical growth in the mean sample of class III malocclusions tends to be greater in the Lower Gonial angle parameter. The direction of horizontal growth 
in the mean class III malocclusion sample tends to be greater in the SNB and Upper Gonial angle parameters. The direction of vertical growth in the mean sample of class I malocclusions tends to be greater in the Facial Axis and Lower Gonial Angle parameters. This provides information that hyperdivergence can occur in class I and class III malocclusions. ${ }^{14}$

The Saddle Angle in class III samples still in the normal range. Saddle Angle is the center of sagittal growth in spheno-occipital syncondrosis. The normal saddle angle shows the normal location of the mandibular fossa, ${ }^{19}$ so the average class III malocclusion Javanese ethnic sample in UNAIR Dental Hospital that sagittal dysplasia is not caused by the position of the mandibular fossa.

Orthodontic treatment to prevent the growth direction above can be done at the age of growth, chin cup is quite recommended because it more restrains the posterior growth of the condyle and prevents the rotation of growth in the angulus. $^{8,9,12}$ In extreme cases, surgery is performed by doing BSSO because it can reduce Upper and Lower Gonial Angle by allowing rotation as well as mandibular setback. ${ }^{20}$

A good diagnosis of sagittal dysplasia is not enough to detect only anteroposterior disharmony, but it is better to be able to detect vertical problems. ANB since it was discovered by Riedell, has become a popular sagittal dysplasia parameter until now. However, in cases with vertical problems such as mandibular rotation, ANB is biased and inaccurate. ${ }^{14,16}$

Witts Appraisal involving the occlusal plane and the AB plane. Witts Appraisal provides a solution to the lack of ANB as a sagittal dysplasia parameter. Witts Appraisal has been a good parameter of jaw disharmony to the present. ${ }^{14,16,21}$ However, in the case of hyperdivergence (large FMA) Witts Appraisal becomes less accurate. Modifications of the Witts appraisal are required by using the "Normal" Occlusal Plane as the reference plane. ${ }^{21}$

In this study, the OP-AB angle provides significant results to distinguish class I and class III malocclusions. The occlusal plane is a true sagittal relationship parameter ${ }^{14,16}$ combined with the AB plane should be able to give a perspective of jaw disharmony like Witts Appraisal. There is no single parameter in cephalometric analysis that is able to provide an absolute perspective of interpretation. ${ }^{14,18,21}$

Cephalometry analysis is a matter of geometry of planes and spaces. The ability to think visually is a very important factor needed to solve geometry problems. Visualization is the ability to see and understand problem situations. The position of the angle and plane parameters in cephalometry should be able to provide visual thinking. ${ }^{22}$ The ANB angle is more popular and used until today because it provides better imaginary visualization than the Witts appraisal, although the Witts appraisal is an excellent jaw disharmony parameter. $^{12,22}$

The OP-AB angle is expected to be able to provide imaginary visualization as well as being a good jaw disharmony parameter. Thus, the OP-AB angle becomes visual thinking which is expected to provide a visualization role that helps in solving orthodontic problems. The OP-AB angle is quite interesting and it is important to continue to be investigated to support the diagnosis and treatment plan. ${ }^{12,22}$

\section{Conclusion}

The OP-AB angle plays an important role in determining class III malocclusion diagnosis. The OP-AB angle is a parameter that can distinguish between class III with class I malocclusion. The OP-AB angle has a strong correlation with vertical and horizontal growth directions.

\section{Disclosure}

The authors report no conflict of interest in this work.

\section{References}

1. Burns NR, Musich DR, Martin C, Razmus T, Gunel E, Ngan P. Class III camouflage treatment: what are the limits? Am J Orthod Dentofacial Orthop. 2010;137:1-13.

2. Mi KB, Kang B, Hong G, Baek S. Prognosis prediction for class III malocclusion treatment by feature wrapping method. Angle Orthod. 2009;79(4):683-691. doi:10.2319/071508-371.1

3. Dwight H. Prevalence of angle class III malocclusion: a systematic review and meta analysis. Open J Epidemiol. 2012;2(4):75-82.

4. Fang N, Yinzhong D. Camouflage treatment in adult skeletal class III case by extraction of two lower premolars. Korean J Orthod. 2010;40 (5):349-357. doi:10.4041/kjod.2010.40.5.349

5. Jin-le L, Chen F, Chen S. Changes of occlusal plane inclination after orthodontic treatment in different dentoskeletal frames. Prog Orthod. 2014;15:41. doi:10.1186/s40510-014-0041-1

6. Parente E, Lazerda G, Silvares MG. Surgical manipulation of the occlusal plane in classical III deformities: 5 features to help planning. Open J Stomatol. 2014;4(5)238-242.

7. Ju BE, Kwon HJ, Kwon O. Changes in longitudinal craniofacial growth in subjects with normal occlusions using the ricketts analysis. Korean J Orthod. 2014;44(2):77-87. doi:10.4041/kjod. 2014.44.2.77

8. Ngan P, Moon W. Evolution of class III treatment in orthodontics. Am J Orthod Dentofacial Orthop. 2015;4(12):22-33.

9. Edlira Z, Chaudhari PK, Sharan J, Dhingra K, Tiwari N. Developing class III malocclusions: challenges and solutions. Clin Cosmet Investig Dent. 2018;10:99-116. doi:10.2147/CCIDE.S134303

10. Vukusic N, Lapter M, Muretic Z. Change in the inclination of the occlusal plane during craniofacial growth and development. Coll Antropol. 2000;24:145-150.

11. Motonori S, Motoyoshi M, Hirabayashi M, Kouhei H, Mitsui N, Shimizu N. Inclination of the occlusal plane is associated with the direction of the masticatory movement path. Eur J Orthod. 2007;29:21-24. doi:10.1093/ejo/cj1036 
12. Senisik NE, Hasipek S. Occlusal cant: etiology, evaluation, and management. Turkish J Orthod. 2015;27(4):174-180.

13. Profit WR, Fields HW. Contemporary orthodontics, 6th, Mosby. Philadelphia. 2019;145-294.

14. Jacobson A, Jacobson R. Radiographic Cephalometry from Basic to 3-D Imaging. 2nd ed. Quintensence Publishing Co Inc Chicago; 2006:137-144.

15. Ricketts RM. The influence of orthodontics treatment on facial growth and development. Angle Orthod. 1960;30:103.

16. Kumar V, Sundareswaran S. Cephalometric assessment of sagittal dysplasia: a review of twenty one methods. J Indian Orthod Soc. 2014;48(1):33-41.

17. Jae HY, Huh KH, Hong KK, et al. Constancy of the angle between the Frankfort horizontal plane and the sella-nasion line A nine-year longitudinal study. Angle Orthod. 2014;84(2):286-289. doi:10.2319/062013464.1
18. Shamlan MA, Aldreess AM. Hard and soft tissue correlation in facial profiles: a canonical correlation study. Clin Cosmet Investig Dent. 2015;9-14.

19. Yassir A. Saddle angle and its relationship with maxillary and mandibular lengths. Iraqi Orthod J. 2009;5:14-16.

20. Reyneke JP. Essentials of Orthognathic Surgery. Quintessence Publishing Co Inc Chicago; 2003:188-213.

21. Hayes JL. A modification of the Wits appraisal is proposed for patients with high FMA or with long lower face height a supplementary assessment. Orthod Pract. 2018;9(2):10-20.

22. Ferrara V, De Santis S, Silvestri A, Staffoli C. Art and medicine: from anatomic studies to visual thinking strategies. Senses Sci. $2015 ; 40-44$

\section{Publish your work in this journal}

Clinical, Cosmetic and Investigational Dentistry is an international, peer-reviewed, open access, online journal focusing on the latest clinical and experimental research in dentistry with specific emphasis on cosmetic interventions. Innovative developments in dental materials, techniques and devices that improve outcomes and patient satisfaction and preference will be highlighted. The manuscript management system is completely online and includes a very quick and fair peer-review system, which is all easy to use. Visit http://www.dovepress.com/testimonials.php to read real quotes from published authors. 